\title{
Liver enzyme trends in patients taking uninterrupted metformin before and after coronary surgery
}

\author{
Rakan I. Nazer ${ }^{1}$, Mohammed F. Abalhassan ${ }^{1}$, Khalid A. Alburikan ${ }^{1,2}$ \\ ${ }^{1}$ Department of Cardiac Science, King Fahad Cardiac Center, College of Medicine, ${ }^{2}$ Department of Clinical Pharmacy, College of Pharmacy, King \\ Saud University, Riyadh, Kingdom of Saudi Arabia \\ Contributions: (I) Concept and design: RI Nazer; (II) Administrative support: K Alburikan; (III) Provision of study materials or patients: RI Nazer; (IV) \\ Collection and assembly of data: M Abalhassan; (V) Data analysis and interpretation: All authors; (VI) Manuscript writing: All authors; (VII) Final \\ approval of manuscript: All authors. \\ Correspondence to: Rakan I. Nazer, MD. 3642 King Saud University, Riyadh 12372-7143, Kingdom of Saudi Arabia. Email: raknazer@ksu.edu.sa.
}

Background: Metformin is an oral antidiabetic agent belonging to the biguanide class of antidiabetics. Possible novel applications for metformin in cardiovascular disease might exist. The aim of this study was to verify a possible association between pre-operative metformin administration and protection against ischemia-induced liver injury in diabetic patients undergoing coronary artery bypass grafting (CABG) surgery.

Methods: A retrospective case-control series was conducted at a single center. Two hundred consecutive diabetic patients underwent isolated on-pump CABG during a 12-month span (July 2015 - July 2016). Metformin was uninterrupted in patients who took this drug prior to CABG; 68 patients were metformin users (34\%) while 132 patients were taking other antidiabetic agents (66\%). Liver enzymes and other organ markers were consecutively recorded daily for 7 days after surgery and expressed as medians with interquartile range (IQR).

Results: Both the metformin and non-metformin group of patients had similar pre-operative demographic characteristics. The median (IQR) post-operative cardiac enzyme creatinine kinase (CK) MB fraction was significantly lower in the metformin group [46.4 U/L (35.8-66.5) vs. 66.5 U/L (44-94.5), P=0.005]. Total bilirubin $[0.58(0.48-0.82) \mathrm{mg} / \mathrm{dL}$ vs. $0.67(0.56-0.95) \mathrm{mg} / \mathrm{dL}, \mathrm{P}=0.021]$, the transaminase aspartate aminotransferase (AST) [32.5 U/L (25.0-42.0) vs. 37.5 U/L (28.5-56), $\mathrm{P}=0.011]$, the transaminases alanine aminotransferase (ALT) [48.5 U/L (40.0-64.0) vs. 57.0 U/L (44.0-77.0), $\mathrm{P}=0.040]$ and lactate dehydrogenase (LDH) [320.0 U/L (273.5-367.2) vs. 356.5 U/L (289.5-427), $\mathrm{P}=0.014]$ were significantly lower in the metformin group. No differences were noted in clinical outcomes.

Conclusions: In this limited retrospective study, the diabetic patients who took metformin before and after undergoing CABG appeared to have a reduced post-operative surge in the total bilirubin and transaminase liver enzymes. Metformin's role in mitigating oxidative stress in liver cells might explain this observation. Further experimental studies are warranted to verify this possible effect.

Keywords: Metformin; coronary artery bypass grafting (CABG); liver injury; diabetes

Submitted Mar 26, 2018. Accepted for publication May 09, 2018.

doi: 10.21037/cdt.2018.05.04

View this article at: http://dx.doi.org/10.21037/cdt.2018.05.04

\section{Introduction}

Metformin is the most commonly prescribed antidiabetic agent used in type 2 diabetes. Metformin's precursor compound, guanidine, can be traced back to medieval times. It was used as a herbal remedy derived from the plant Galega officinalis (French lilac) (1). The herb was thought to relieve what we now know are symptoms related to diabetes. Metformin belongs to the biguanides family of 
antidiabetic agents and is a synthetic analog of guanidine. Like its precursor agents, metformin possesses antidiabetic properties with few side-effects $(2,3)$. The incidence of lactic acidosis related to metformin use remains low with a reported average of $2-7$ cases per 100,000 patientyears (4). Despite the wide use of metformin as a first-line oral antidiabetic treatment for type 2 diabetes, its exact mechanism of action is yet to be understood $(3,5,6)$. Much of its antidiabetic effect has been largely attributed to the suppression of gluconeogenesis in the liver, as well as the reduction of glucose absorption in the digestive tract and the increase of muscle and fatty tissue sensitivity to insulin. Metformin also has beneficial effects on lipid metabolism, fibrinolysis, and endothelial function $(1,5,7)$. Independent from its glucose-lowering effect, metformin monotherapy has been linked to favorable pleiotropic actions in the treatment of cardiovascular disease, cancer, and aging $(1,3,8,9)$.

A systemic inflammatory reaction is generally observed in all patients after cardiac surgery (10). This is the result of surgical trauma, the initiation of cardio-pulmonary bypass $(\mathrm{CPB})$, and heart/lung ischemia-reperfusion injuries. Much of this is manifested in a notable early transient surge of biomarkers related to the heart, kidneys, liver, muscles, and brain $(10,11)$. The liver is particularly prone to transient hepatocellular damage after on-pump coronary artery bypass grafting (CABG) (12). This is most likely due to splanchnic hypoperfusion during $\mathrm{CPB}$, which is usually marked with an increase in liver alanine aminotransferase (ALT) enzyme levels (13). In animal experiments, metformin has been shown to significantly reverse hepatic steatosis, inflammation, and ALT abnormalities (14-16). The aim of the present retrospective observational study was to determine whether pre-operative metformin administration results in a more attenuated surge in liver biomarkers after on-pump CABG in patients with type 2 diabetes. The findings will help elucidate early clinical applications for a possible liver-protective effect of metformin in humans.

\section{Methods}

After obtaining the approval from our local institutional research ethics committee, an electronic registry for all cardiac procedures performed at King Fahad Cardiac Center was interrogated for patients who underwent isolated on-pump CABG between July 2015 and July 2016 (a 12-month period). One year prior to data collection, our unit adopted a policy for uninterrupted oral administration of metformin until the day of surgery, with an early reinitiation soon after extubation (in 2-3 hours), for all patients with diabetes who were taking metformin prior to surgery (17). Prior to surgery, $70 \%$ of the patients took $500 \mathrm{mg}$ of metformin twice/day, $20 \%$ took $850 \mathrm{mg}$ twice/day, and $10 \%$ took 1,000 $\mathrm{mg}$ twice/day (mean duration, $26 \pm 9$ months). Critically ill patients in shock and those with acute kidney or liver injury were exempt from this policy. Contraindications to metformin therapy included renal dysfunction (creatinine clearance $\leq 30 \mathrm{~mL} / \mathrm{min}$ ) and the use of two or more nephrotoxic medications. This new strategy was supported by contemporary evidence indicating the safety of metformin with a better glycemic control when combined with other anti-hyperglycemic regimens in the post-operative period (18-22).

Individual patient consent was waived and all relevant demographic, clinical, and laboratory data were retrospectively collected from the medical charts, intensive care unit (ICU) flow sheets, and electronic laboratory records. All patients underwent CABG under general anesthesia. Median sternotomy was performed and CPB was initiated via central cannulation of the aorta and right atrium. The systemic body temperature was cooled to $32{ }^{\circ} \mathrm{C}$ and the heart was arrested after applying a crossclamp using cold blood potassium cardioplegia. The left internal mammary artery was grafted to the left anterior descending artery in 196 patients (98\%). A segment of the greater saphenous vein was used to graft other targets. Intraoperatively, blood glucose levels, activated clotting time (ACT), electrolytes, and blood gases were measured every 30 minutes. Once the patient arrived in the ICU, the blood glucose level was measured every hour. All patients were subjected to a hospital-wide standardized glycemic control protocol using continuous regular insulin infusion. The aim of this protocol was to maintain the blood glucose level between $100-149 \mathrm{mg} / \mathrm{dL}$ in the operating room and ICU. When the blood glucose level exceeded $248 \mathrm{mg} / \mathrm{dL}$, additional blouses of regular insulin were administered according to a sliding scale. When blood glucose level reached 59-99 $\mathrm{mg} / \mathrm{dL}$, insulin infusion was reduced by $50 \%$. When blood glucose level was $<59 \mathrm{mg} / \mathrm{dL}$, insulin infusion was stopped and $50 \mathrm{~mL}$ of D50 dextrose bolus was administered. After surgery, all patients spent at least 24 hours in the ICU; thereafter, they were transferred to a monitored bed until the day of discharge.

Total creatinine kinase (CK) (U/L), CK-MB fraction (U/L), and high sensitivity Troponin I $(\mu \mathrm{g} / \mathrm{L})$, were recorded in terms of peak levels during the first two days 
after surgery. Fasting blood sugar $(\mathrm{mg} / \mathrm{dL})$, serum creatinine $(\mathrm{mg} / \mathrm{dL})$, total bilirubin $(\mathrm{mg} / \mathrm{dL})$, alkaline-phosphatase (ALP) (U/L), ALT (U/L), aspartate aminotransferase (AST) (U/L), gamma-glutamyl transferase (GGT) (U/L), and lactate dehydrogenase $(\mathrm{LDH})(\mathrm{U} / \mathrm{L})$ levels were recorded prior to surgery and measured daily for up to 7 days after surgery. For every patient in the series, each liver enzyme was expressed as a median and interquartile range (median, IQR) for the first 7 days after surgery. Serum lactic acid levels $(\mathrm{mg} / \mathrm{dL})$ were measured on an hourly basis in the ICU (via a blood gas analysis) for 24 hours after surgery and were expressed as peak lactic acid.

\section{Statistics}

Statistical analyses were performed using SPSS 16.0 software (SPSS Inc., Chicago, IL, USA). Continuous variables are expressed as means \pm standard deviations. Continuous variables not normally distributed were expressed as medians with IQR. Group differences were evaluated using t-tests for continuous variables. Continuous variables not normally distributed were subjected to a Mann-Whitney $U$ test. Categorical variables were tabulated in $2 \times \mathrm{n}$ tables and group differences were evaluated using the $\chi^{2}$-test or Fisher exact test where one or more cells has an expected frequency of five or less. Clinically important variables or those with a $\mathrm{P}<0.1$ in univariable regression analyses were entered into a multi-step logistic regression model. The results are expressed as odds ratios (OR), 95\% confidence interval (95\% CI), and P values. A two-tailed $\mathrm{P}$ value $<0.05$ was considered statistically significant.

\section{Results}

After excluding all patients who had CABG combined with valve or non-valve procedures, we identified a total of 277 patients who underwent isolated on-pump CABG. Among these, 200 patients (72\%) had type 2 diabetes prior to surgery. At the time of CABG, 68 patients (34\%) were taking metformin and 132 patients were taking other antidiabetic agents (66\%). Table 1 summarizes the baseline characteristics comparing both groups with no significant difference in age, sex, or clinical presentation such as myocardial infarction and left ventricular dysfunction. Prior to surgery, metformin users had a slightly higher glycosylated hemoglobin (HbA1C) level $(8.8 \% \pm 1.6 \%$ vs. $7.8 \% \pm 1.9 \% ; \mathrm{P}=0.001)$, but a significantly lower serum creatinine level $(0.96 \pm 0.24$ vs. $1.10 \pm 0.27 \mathrm{mg} / \mathrm{dL} ; \mathrm{P}=0.006)$ compared to that in metformin non-users (Table 1). Metformin users started with a higher fasting blood sugar level compared to that in metformin non-users $(196 \pm 81 \mathrm{vs}$. $151 \pm 68 \mathrm{mg} / \mathrm{dL} ; \mathrm{P}<0.001)$.

During surgery, metformin users and non-users had similar CPB times, number of distal coronary grafts and ventilation times (Table 2). After surgery, the mean postoperative cardiac index was slightly but significantly lower in metformin non-users compared to that in metformin users (2.9 \pm 0.8 vs. $\left.2.6 \pm 1.1 \mathrm{~L} / \mathrm{M}^{2} ; \mathrm{P}=0.038\right)$, which was associated with a higher use of inotropic agents administered in the non-users (19.1\% vs. 33.3\%; $\mathrm{P}=0.047)$. The peak lactic acid level in the first 24 hours after surgery was significantly higher in non-metformin users compared to that in metformin users $(49.5 \pm 24 \mathrm{mg} / \mathrm{dL}$ vs. $65.8 \pm 35 \mathrm{mg} / \mathrm{dL}$; $\mathrm{P}<0.001)$. With the exception of more pulmonary-related complications (i.e., atelectasis, lung collapse, pneumonia, and effusions) in non-users compared to that in metformin users $(11.8 \%$ vs. $25.0 \%$; $\mathrm{P}=0.028)$, the clinical endpoints (including death, myocardial infarction, stroke, renal failure, infections, and atrial fibrillation) were similar. In addition, the post-operative use of standard cardiac medications in CABG was similar in the two groups (Table 2).

The (median, IQR) peak postoperative cardiac enzyme levels for CK-MB [46.4 U/L (35.8-66.5) vs. 66.5 U/L (44-94.5), $\mathrm{P}=0.005]$ was significantly lower in the metformin group. Troponin I was similar in the two groups [1.1 U/L (0.57-1.6) vs. $1.3 \mathrm{U} / \mathrm{L}(0.3-2.6), \mathrm{P}=0.332]$ (Table 3). The postoperative median serum creatinine level was significantly higher in non-users compared to that in metformin users $[0.99(0.85-1.15) \mathrm{mg} / \mathrm{dL} v s .1 .10$ $(0.97-1.32) \mathrm{mg} / \mathrm{dL} ; \mathrm{P}=0.001]$. The median post-operative total bilirubin $[0.58(0.48-0.82)$ vs. $0.67(0.56-0.95) \mathrm{mg} / \mathrm{dL}$; $\mathrm{P}=0.021$ ], transaminase liver enzyme levels for ALT [48.5 U/L (40.0-64.0) vs. 57.0 U/L (44.0-77.0); $\mathrm{P}=0.040]$, transaminase liver enzyme AST [32.5 U/L (25.0-42.0) vs. $37.5 \mathrm{U} / \mathrm{L}$ (28.5-56); $\mathrm{P}=0.011$ ] (Figures 1-3) all were significantly less post-operatively in the metformin users (Table 3). The difference between the post-operative and baseline levels for the same liver enzyme was also significantly less pronounced in the metformin users (total bilirubin 0.22 vs. $0.30 \mathrm{mg} / \mathrm{dL} ; \mathrm{P}=0.007$, ALT 5.5 vs. $13 \mathrm{U} / \mathrm{L} ; \mathrm{P}=0.002$, AST 9.0 vs. $16.7 \mathrm{U} / \mathrm{L}$; $\mathrm{P}=0.023)$. The median serum LDH level was significantly higher in the non-metformin users compared to that in the metformin users [320.0 U/L (273.5-367.2) vs. $356.5 \mathrm{U} / \mathrm{L}$ (289.5-427), $\mathrm{P}=0.014]$. Prior to surgery, the baseline measured enzymes were similar (Table 3). A sub- 
Table 1 Baseline characteristics

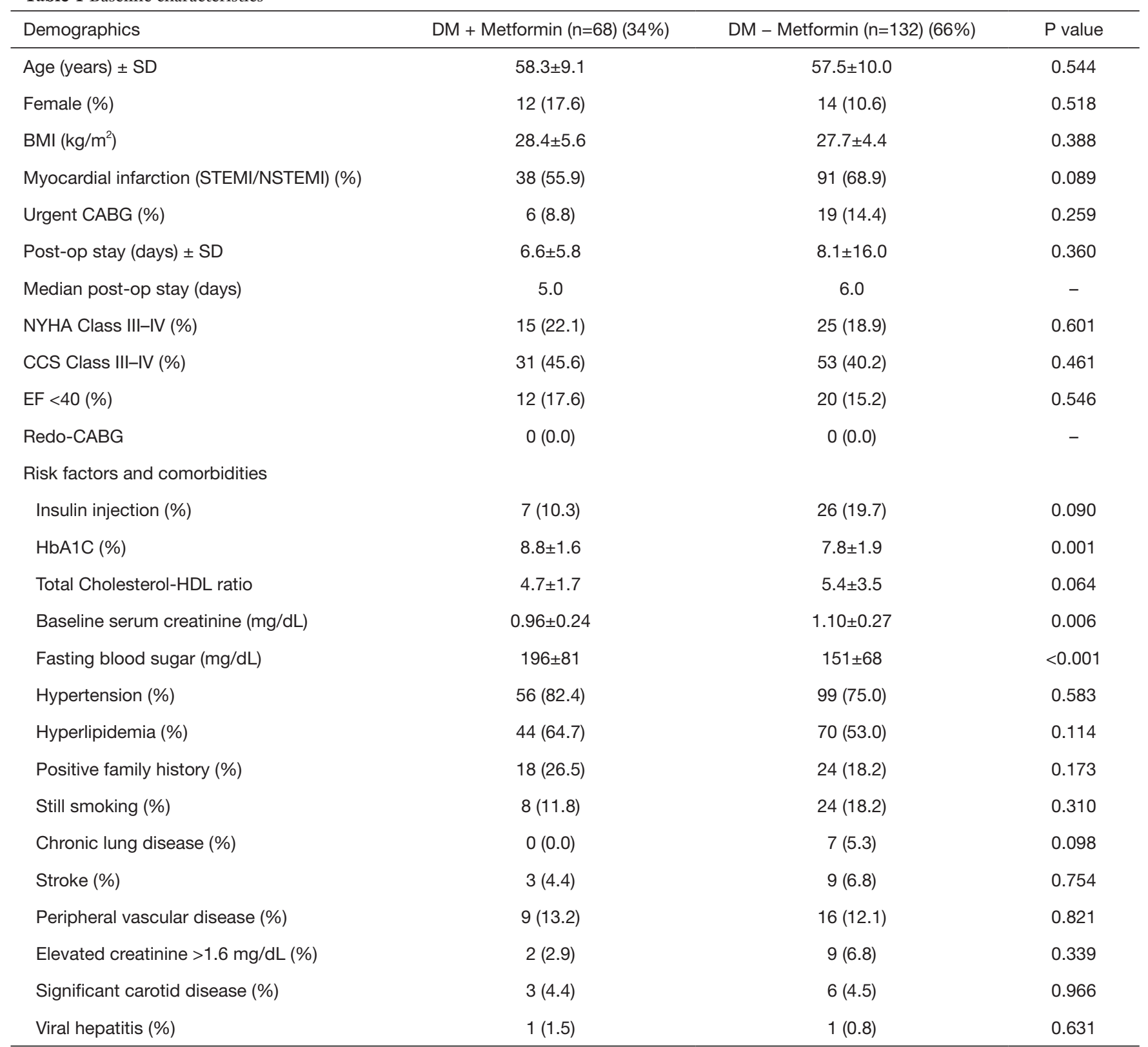

DM, diabetes mellitus; BMI, body mass index; STEMI, ST-elevation myocardial infarction; NSTEMI, non-STEMI; CABG, coronary artery bypass grafting; NYHA, New York Heart Association; CCS, Canadian cardiac society, EF, ejection fraction; HbA1C, hemoglobin A1c; HDL; high-density cholesterol

group analysis of the cardiac, kidney and liver enzymes of the same 68 metformin users (53\%) and the originally excluded 77 non-diabetic patients (47\%) who had isolated CABG from the same series, revealed a uniformly significantly lower enzyme levels after CABG in the metformin users (Table 4).

The multivariable logistic regression model (Table 5) examining the predictors of the ALT liver transaminase enzyme level equal to or greater than a median of $60 \mathrm{U} / \mathrm{L}$ in the first 7 days after surgery-roughly double the upper normal for our local population, identified pre-operative use of metformin as a protector (OR, $0.42 ; 95 \%$ CI, 0.22-0.78; $\mathrm{P}=0.006)$ and the administration of vasopressors as a hazard (OR, 2.01; 95\% CI, 1.05-3.83; P=0.034) (Goodness of fit: 
Table 2 Post-operative outcomes

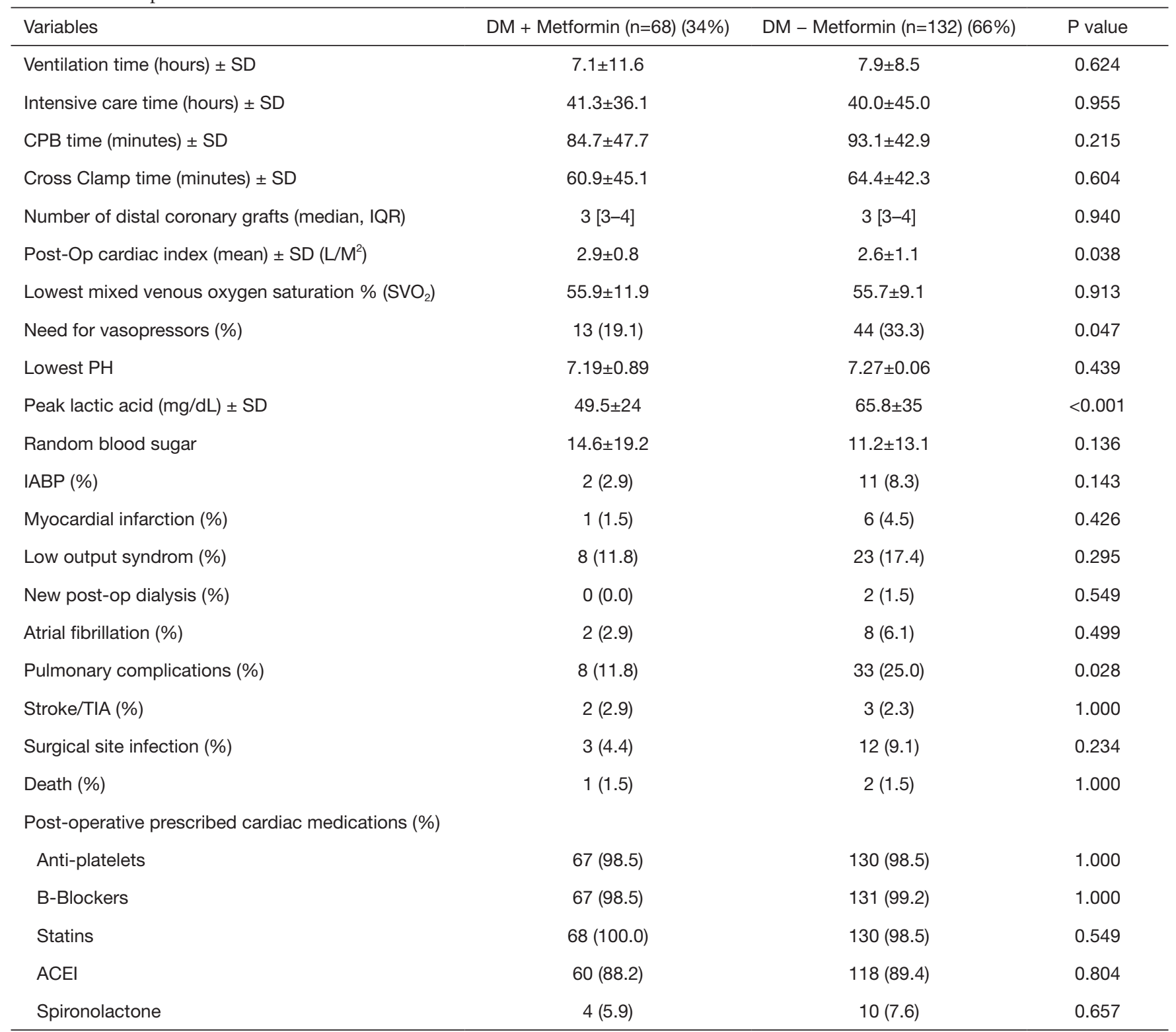

DM, diabetes mellitus; CPB, cardio-pulmonary bypass; $\mathrm{SVO}_{2}$, mixed venous oxygen saturation; IABP, intra-aortic balloon pump, TIA, transient ischemic attack; ACEI, angiotensin converting enzyme inhibitors; IQR, interquartile range.

Hosmer-Lemeshow test $\mathrm{P}$ value is 0.598 and omnibus test $\mathrm{P}$ value $<0.001)$.

\section{Discussion}

Metformin, a drug commonly used to treat patients with type 2 diabetes, might have potentials beyond controlling elevated blood sugar. Studies have identified multiple molecular pathways underlying the possible protective mechanisms of metformin in diabetes, cardiovascular disease, ageing, and cancer. These include a myriad of pathways leading to reduced insulin-like growth factor signaling; inhibition of the mechanistic target of rapamycin (mTOR); inhibition of mitochondrial complex-1, causing a reduced production of reactive oxygen species (ROS); and the activation of AMP-activated kinase, which inhibits gluconeogenesis in the liver $(1,5,6,23)$. Thus, metformin may exert protective effects against oxidative 
Table 3 Post-operative laboratory measurements (median, IQR)

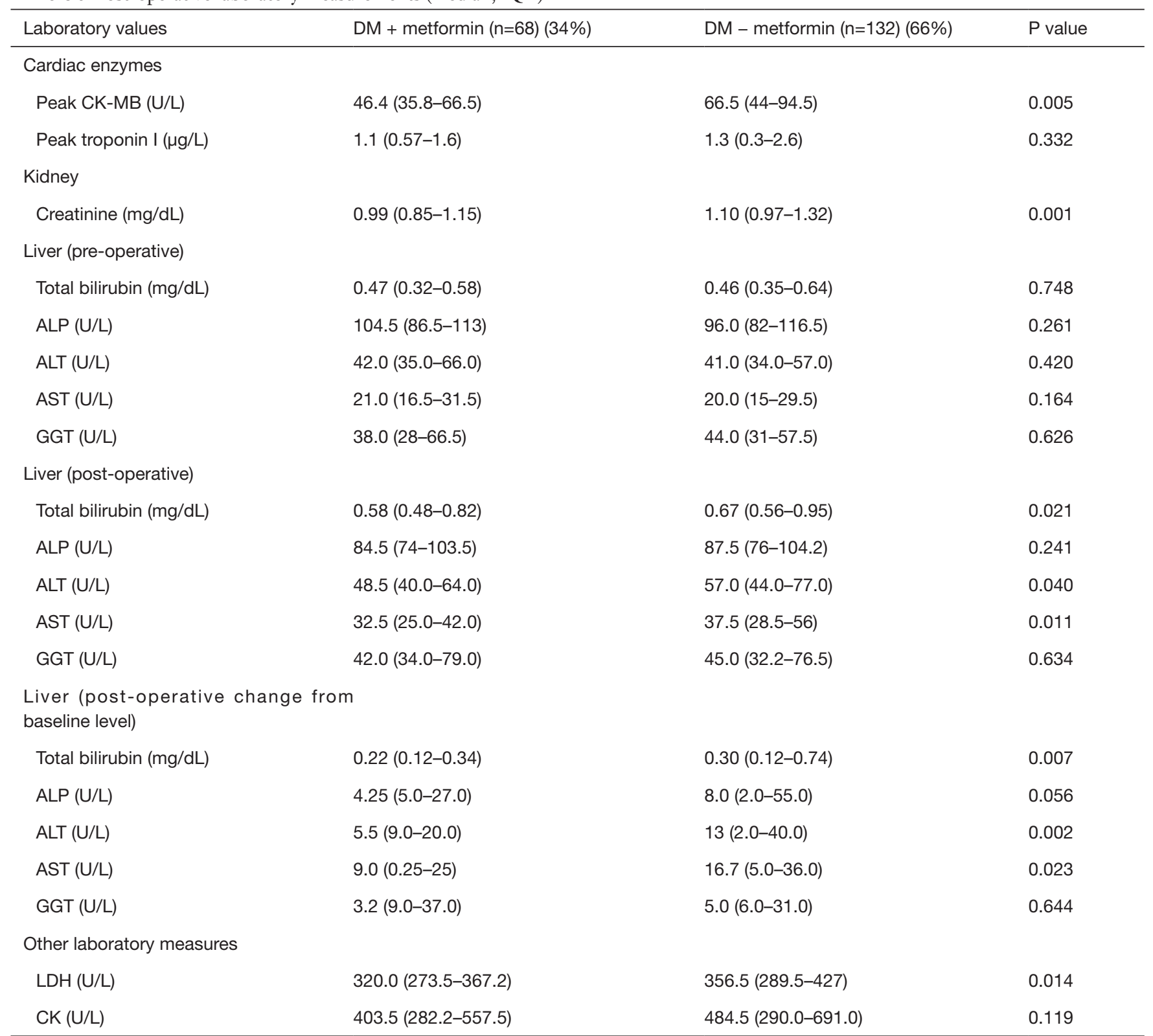

DM, diabetes mellitus; CK creatinine kinase; CK-MB CK MB fraction; ALP, alkaline-phosphatase; ALT, alanine aminotransferase; AST, aspartate aminotransferase; GGT, gamma-glutamyl transferase; LDH, lactate dehydrogenase.

stress via some of these molecular pathways. In the liver, metformin maintains glutathione (an important antioxidant in mitochondrial hepatocytes) in a reduced state. Maintaining hepatic mitochondrial glutathione in a reduced state is essential for cell survival (24). In both animal and human models, hypothermic CPB is associated with subtle hemodynamic changes in the mucosal and hepatic sinusoidal microcirculations, which contribute to splanchnic hypoperfusion and ischemia (25). This is further aggravated by postoperative increased oxygen demand, a low cardiac output state, and the use of vasoactive agents. This cascade of events can inflect visceral organ injury, manifesting in a recognized pattern of liver, kidney, and pancreas biomarker surge within the first $2-3$ days after surgery $(11,12,26)$. The 


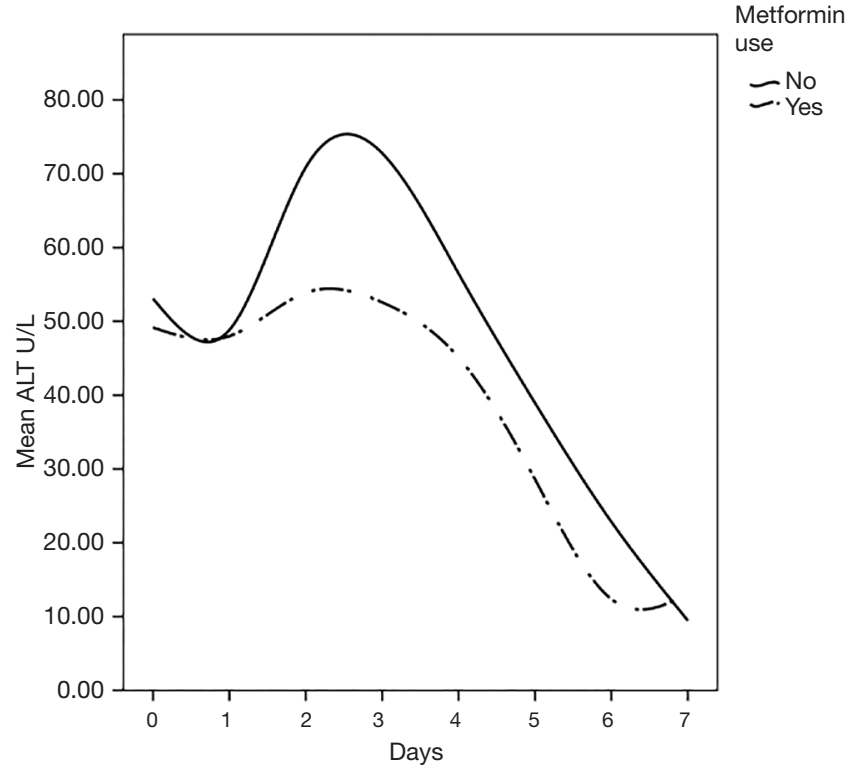

Figure 1 The mean ALT level is shown according to the number of days after surgery. ALT, alanine aminotransferase.

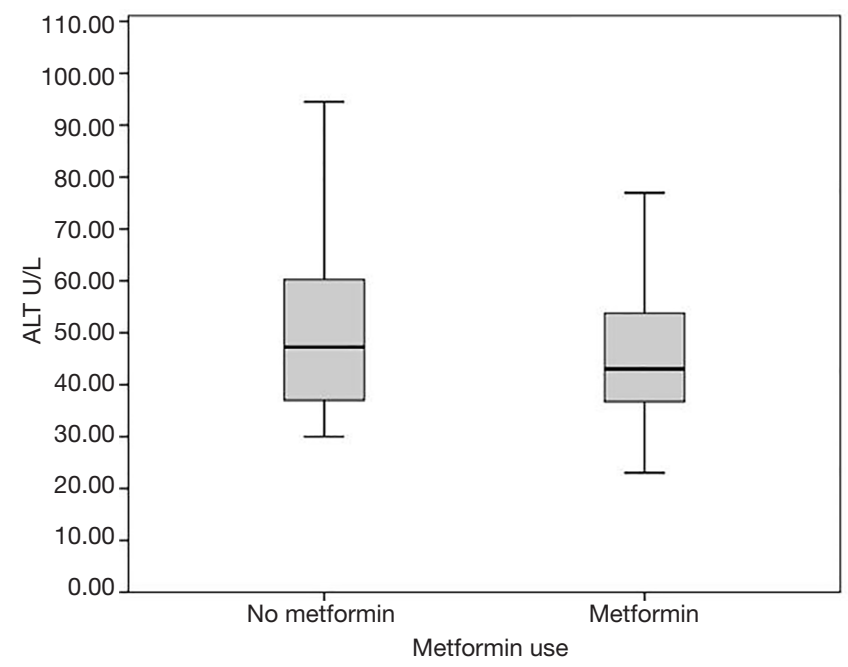

Figure 2 A box-plot comparison of ALT levels based on metformin use is shown. ALT, alanine aminotransferase.

present study demonstrates a clear pattern of hepatocellular injury exhibited through a surge in the liver transaminase enzymes, AST and ALT, as well as total bilirubin, reaching a high peak by the $3^{\text {rd }}$ day after surgery before returning to normal levels by day 7 . While AST is commonly released after CPB, ALT is more specific to ischemia-induced hepatocellular injury (13). Our serial postoperative analysis of liver function demonstrated a significantly reduced

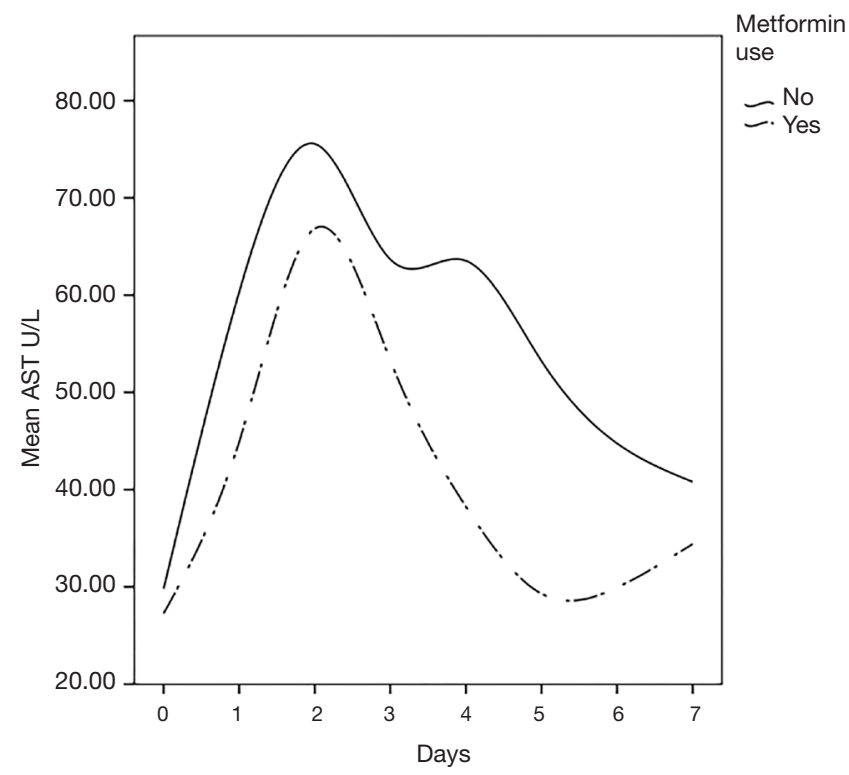

Figure 3 The mean AST level is shown according to the number of days after surgery. AST, aspartate aminotransferase.

transaminase enzyme release in patients who were taking metformin during the pre- and post-operative periods; this was also associated with a more attenuated transaminase surge pattern after CABG compared to those who did not take metformin pre-operatively. At this point, the relationship of metformin use and reduced liver enzyme surge is merely an association not causation.

All patients in the present study had type 2 diabetes prior to surgery as evidenced by the high glycosylated HbA1C and fasting blood sugar levels. Those who took metformin tended to have slightly higher BMIs and significantly higher blood sugar levels. In addition, the serum creatinine level before surgery was significantly less in those who took metformin prior to surgery. This is probably a reflection of the common general practice of not prescribing metformin for those with impaired kidney function. Overall, both groups were similar in terms of risk factors and characteristics, including the degree of left ventricular function impairment. In the postoperative period, there was a subtle, yet statistically significant, trend for a lower cardiac index in metformin non-users over the first 24 hours after surgery $\left(2.8 v s .2 .6 \mathrm{~L} / \mathrm{M}^{2}\right)$. This might reflect a higher (but not statistically significant) fraction of patients who needed urgent CABG among the metformin non-users compared to that in the metformin users (14.4\% vs. $8.8 \%)$. This in turn may have led to a significant higher post-operative CK-MB fraction cardiac enzyme leading 
Table 4 Sub-group analysis of metformin users versus non-diabetics from the same series

\begin{tabular}{|c|c|c|c|}
\hline Laboratory values & \multicolumn{2}{|c|}{ Isolated CABG in 145 patients } & $P$ value \\
\hline Blood sugar $(\mathrm{mg} / \mathrm{dL})($ mean $\pm \mathrm{SD})$ & $263 \pm 345$ & $133 \pm 21.6$ & 0.003 \\
\hline \multicolumn{4}{|l|}{ Cardiac enzymes (medians, IQR ) } \\
\hline Peak CK-MB (U/L) & $46.4(35.8-66.5)$ & $79(57.0-110.0)$ & 0.001 \\
\hline \multicolumn{4}{|l|}{ Kidney (medians, IQR ) } \\
\hline Creatinine (mg/dL) & $0.99(0.85-1.15)$ & $1.31(1.14-1.65)$ & $<0.001$ \\
\hline \multicolumn{4}{|l|}{ Liver (post-operative) (medians, IQR) } \\
\hline Total bilirubin (mg/dL) & $0.58(0.51-13.7)$ & $1.11(0.76-1.7)$ & $<0.001$ \\
\hline AST (U/L) & $32.5(25.0-42.0)$ & $70(54.0-129.0)$ & $<0.001$ \\
\hline GGT (U/L) & $42.0(34.0-79.0)$ & $66(46.0-119.0)$ & $<0.001$ \\
\hline \multicolumn{4}{|l|}{ Other laboratory measures } \\
\hline LDH (U/L) & $320.0(273.5-367.2)$ & $494(442.0-574.0)$ & $<0.001$ \\
\hline CK (U/L) & $403.5(282.2-557.5)$ & $1137(842.0-1,495.0)$ & $<0.001$ \\
\hline
\end{tabular}

DM, diabetes mellitus; CK creatinine kinase; CK-MB CK MB fraction; ALP, alkaline-phosphatase; ALT, alanine aminotransferase; AST, aspartate aminotransferase; GGT, gamma-glutamyl transferase; LDH, lactate dehydrogenase.

Table 5 Regression analysis for predicting a median post-operative ALT of $\geq 60$ U/L in 200 patients with diabetes undergoing isolated on-pump CABG in 1 year

\begin{tabular}{|c|c|c|c|c|c|c|}
\hline Variables & \multicolumn{3}{|c|}{ Univariable } & \multicolumn{3}{|c|}{ Multivariable stepwise } \\
\hline Pre-operative administration of metformin & 0.40 & $0.22-0.76$ & 0.005 & 0.42 & $0.22-0.78$ & 0.006 \\
\hline Need for multiple vasopressors & 1.92 & $1.9-3.7$ & 0.051 & 2.01 & $1.05-3.83$ & 0.034 \\
\hline Post-operative low output syndrome & 1.68 & $0.74-3.8$ & 0.213 & - & - & - \\
\hline Renal dysfunction pre-operatively & 1.07 & $0.30-3.8$ & 0.915 & - & - & - \\
\hline
\end{tabular}

ALT, alanine aminotransferase; CABG, coronary artery bypass grafting.

to a higher number of vasopressors used in the immediate postoperative period among the non-users. Consequently, due to low cardiac indexes and high vasoactive support in metformin non-users, the postoperative surge in serum lactate, liver transaminase, creatinine, and LDH levels was significantly higher for this group. The use of metformin pre-operatively was predictive for favorable effect against elevated median ALT ( $\geq 60 \mathrm{U} / \mathrm{L})$ [an ALT level of $60 \mathrm{U} / \mathrm{L}$ is roughly double the normal upper limit in the local population (27)]. The use of vasopressors was predictive of an elevated median ALT. Whether metformin directly interacts with liver hepatocytes by making them more resilient to ischemia-induced liver injury has yet to be investigated.

The notion that metformin may potentially harbor liverprotective properties is not entirely new. Earlier studies 
have demonstrated that treatment with metformin reverses fatty liver disease in animal models. Lin et al. observed that steatosis virtually disappeared from the livers of metformintreated mince, which was also associated with significantly lower levels of serum aminotransferases and alkaline phosphatase (15). Kita et al. demonstrated that an 8-week treatment with metformin prevented and reversed steatosis, inflammation, and fibrosis in non-diabetic steatohepatitis mouse models (14). Recently, Saravi et al., investigated whether metformin reversed oxidative stress-induced liver injury using acetaminophen toxicity in mice and found a significant decrease in serum ALT, AST, ALP , and all proinflammatory markers in the metformin-treated mice (16). The authors attributed this finding to the proposed metformin-inhibiting effects on mitochondrial oxidative stress and ROS production. In humans, evidence from epidemiologic studies suggests that long-term metformin treatment significantly reduces mean transaminase concentration and improves hepatocyte viability in nonalcoholic steatohepatitis $(28,29)$. As part of the Metformin for Non-diabetic Patients with Coronary Heart Disease (CAMERA) study, 173 non-diabetic patients with coronary heart disease were prospectively randomized to either receive metformin $850 \mathrm{mg}$ twice per day or a placebo (30). Although the study showed no difference in the primary outcome measure of carotid intima-media thickness after 18 months, the metformin group had a significantly reduced Y-glutamyl transferase level (but ALT concentrations did not change significantly). The study by El Messaoudi et al. (31) was the first of its kind and, as part of the MetCAB study, 111 non-diabetic patients undergoing elective on-pump CABG were prospectively randomized to receive $500 \mathrm{mg}$ of metformin three times a day or placebo 3 days prior to surgery. Metformin did not impact high-sensitive troponin I levels after CABG, nor did it influence the need for inotropic support or intensive care stay. In addition, the metformin-treated group did not show any evidence of lactic acidosis. The researchers concluded that a shortterm pretreatment with metformin has no effect on postoperative myocardial injury in patients without diabetes undergoing CABG. We have similarly concluded that metformin does not influence the post-operative peak high sensitivity Troponin I but did find a lower CK-MB fraction levels in the metformin group. However, biomarkers released by the liver were not examined in the MetCAB study; thus, it cannot be concluded that metformin has no positive impact on other visceral organs. The benefits of metformin may only manifest in a sicker cohort of patients, such as those with diabetes. In addition, the MetCAB study might have been underpowered to identify subtle changes in cardiac enzymes in such a relatively low-risk population.

The present study has several limitations, including those inherent in any descriptive retrospective analysis. The inability to adjust for multiple confounders can cast doubt on some of the findings of this study. In addition, the sample size was relatively small. Furthermore, sonographic imaging of the liver to rule out any pre-existing liver disease, such as fatty liver, was not conducted. Without liver imaging, it is difficult to determine whether the differences in postoperative aminotransferase enzyme levels are correlated with postoperative structural liver changes or not. Furthermore, although multiple prospective studies have demonstrated the safety of metformin administration in the periprocedural period for patients undergoing CABG $(20,22,31,32)$, it is feared that metformin-associated lactic acidosis might still remain a potential risk for some patients.

In conclusion, it is widely accepted that many patients undergoing on-pump CABG will sustain a transient visceral ischemic injury due to splanchnic hypoperfusion. This injury is further aggravated by a postoperative low cardiac output state and the use of vasoconstrictors. The manifestation of this injury involves a short-lived surge in liver transaminases, especially ALT as it is mostly harbored in the liver hepatocytes. Metformin may play a role in mitigating such effects through biochemical pathways related to the inhibition of the oxidative-stress response in the mitochondria and the replenishment of reduced glutathione in the liver, which acts as its principal antioxidant. Whether metformin truly help protect the liver from injury in CABG warrants further assessment through a carefully constructed randomized clinical trial.

\section{Acknowledgements}

This project was supported by College of Medicine Research Centre, Deanship of Scientific Research, King Saud University.

\section{Footnote}

Conflicts of Interest: The authors have no conflicts of interest to declare.

Ethical Statement: This study was approved by King Saud University College of Medicine Institutional Review Board 
No. E-13-1041. As this was a retrospective study with no direct patient identifiers revealed, individual consent was waived.

\section{References}

1. Pryor R, Cabreiro F. Repurposing metformin: an old drug with new tricks in its binding pockets. Biochem J 2015;471:307-22.

2. Brown JB, Pedula K, Barzilay J, et al. Lactic acidosis rates in type 2 diabetes. Diabetes Care 1998;21:1659-63.

3. Marshall SM. 60 years of metformin use: a glance at the past and a look to the future. Diabetologia 2017;60:1561-5.

4. Lalau JD. Lactic acidosis induced by metformin: incidence, management and prevention. Drug Saf 2010;33:727-40.

5. El Messaoudi S, Rongen GA, Riksen NP. Metformin therapy in diabetes: the role of cardioprotection. Curr Atheroscler Rep 2013;15:314.

6. Rena G, Hardie DG, Pearson ER. The mechanisms of action of metformin. Diabetologia 2017;60:1577-85.

7. Sirvinskas E, Kinduris S, Kapturauskas J, et al. Perioperative use of metformin in cardiac surgery. Medicina (Kaunas) 2010;46:723-9.

8. Heckman-Stoddard BM, DeCensi A, Sahasrabuddhe VV, et al. Repurposing metformin for the prevention of cancer and cancer recurrence. Diabetologia 2017;60:1639-47.

9. Valencia WM, Palacio A, Tamariz L, et al. Metformin and ageing: improving ageing outcomes beyond glycaemic control. Diabetologia 2017;60:1630-8.

10. Levy JH, Tanaka KA. Inflammatory response to cardiopulmonary bypass. Ann Thorac Surg 2003;75:S715-20.

11. Shahbazi S, Panah A, Sahmeddini MA. Evaluation of factors influencing liver function test in on-pump coronary artery bypass graft surgery. Iran J Med Sci 2013;38:308-13.

12. Yamada T, Ochiai R, Takeda J, et al. Off-pump coronary artery bypass attenuates transient hepatocellular damage after myocardial revascularization. J Cardiothorac Vasc Anesth 2005;19:603-7.

13. Ascione R, Talpahewa S, Rajakaruna C, et al. Splanchnic organ injury during coronary surgery with or without cardiopulmonary bypass: a randomized, controlled trial. Ann Thorac Surg 2006;81:97-103.

14. Kita Y, Takamura T, Misu H, et al. Metformin prevents and reverses inflammation in a non-diabetic mouse model of nonalcoholic steatohepatitis. PLoS One 2012;7:e43056.

15. Lin HZ, Yang SQ, Chuckaree C, et al. Metformin reverses fatty liver disease in obese, leptin-deficient mice. Nat Med 2000;6:998-1003.

16. Saeedi Saravi SS, Hasanvand A, Shahkarami K, et al. The protective potential of metformin against acetaminopheninduced hepatotoxicity in BALB/C mice. Pharm Biol 2016;54:2830-7.

17. Nazer RI, Alburikan KA. Metformin is not associated with lactic acidosis in patients with diabetes undergoing coronary artery bypass graft surgery: a case control study. BMC Pharmacol Toxicol 2017;18:38.

18. Mojtahedzadeh M, Jafarieh A, Najafi A, et al. Comparison of metformin and insulin in the control of hyperglycaemia in non-diabetic critically ill patients. Endokrynol Pol 2012;63:206-11.

19. Panahi $Y$, Mojtahedzadeh $M$, Zekeri N, et al. Metformin treatment in hyperglycemic critically ill patients: another challenge on the control of adverse outcomes. Iran J Pharm Res 2011;10:913-9.

20. Baradari AG, Emami Zeydi A, Aarabi M, et al. Metformin as an adjunct to insulin for glycemic control in patients with type 2 diabetes after CABG surgery: a randomized double blind clinical trial. Pak J Biol Sci 2011;14:1047-54.

21. van der Horst IC, Nijsten MW. Metformin in cardiac surgery: high expectations. Lancet Diabetes Endocrinol 2015;3:581-2.

22. Ghods K, Davari H, Ebrahimian A. Evaluation of the effect of metformin and insulin in hyperglycemia treatment after coronary artery bypass surgery in nondiabetic patients. Ann Card Anaesth 2017;20:427-31.

23. Barzilai N, Crandall JP, Kritchevsky SB, et al. Metformin as a Tool to Target Aging. Cell Metab 2016;23:1060-5.

24. Poon MK, Chiu PY, Mak DH, et al. Metformin protects against carbon tetrachloride hepatotoxicity in mice. J Pharmacol Sci 2003;93:501-4.

25. Hessel EA 2nd. Abdominal organ injury after cardiac surgery. Semin Cardiothorac Vasc Anesth 2004;8:243-63.

26. Meng QH, Zhu S, Sohn N, et al. Release of cardiac biochemical and inflammatory markers in patients on cardiopulmonary bypass undergoing coronary artery bypass grafting. J Card Surg 2008;23:681-7.

27. Al-hamoudi W, Ali S, Hegab B, et al. Revising the upper limit of normal for levels of serum alanine aminotransferase in a Middle Eastern population with normal liver histology. Dig Dis Sci 2013;58:2369-75.

28. Marchetti P, Gregorio F, Benzi L, et al. Diurnal pattern of plasma metformin concentrations and its relation to metabolic effects in type 2 (non-insulin-dependent) diabetic patients. Diabete Metab 1990;16:473-8. 
29. Ratziu V. Novel Pharmacotherapy Options for NASH. Dig Dis Sci 2016;61:1398-405.

30. Preiss D, Lloyd SM, Ford I, et al. Metformin for nondiabetic patients with coronary heart disease (the CAMERA study): a randomised controlled trial. Lancet Diabetes Endocrinol 2014;2:116-24.

31. El Messaoudi S, Nederlof R, Zuurbier CJ, et al. Effect of metformin pretreatment on myocardial injury during

Cite this article as: Nazer RI, Abalhassan MF, Alburikan KA. Liver enzyme trends in patients taking uninterrupted metformin before and after coronary surgery. Cardiovasc Diagn Ther 2018;8(4):469-479. doi: 10.21037/cdt.2018.05.04 coronary artery bypass surgery in patients without diabetes (MetCAB): a double-blind, randomised controlled trial. Lancet Diabetes Endocrinol 2015;3:615-23.

32. Baradari AG, Habibi MR, Khezri HD, et al. Does highdose metformin cause lactic acidosis in type 2 diabetic patients after CABG surgery? A double blind randomized clinical trial. Heart Int 2011;6:e8. 